\title{
Continuing Education Model for Nurses Regarding Substance Use Disorder
}

\author{
Natalie R. Perry ${ }^{\star}$ and Ilana R Azulay Chertok
}

School of Nursing, College of Health Sciences and Professions, Ohio University, Athens, Ohio, USA

"Corresponding author: Natalie R. Perry, School of Nursing, College of Health Sciences and Professions, Ohio University, Athens, Ohio, USA, Tel: + 3046962639; Email: dgmnatalie24@gmail.com

Received date: August 25, 2018; Accepted date: August 29, 2018; Published date: September 05, 2018

Copyright: (c) 2018 Perry NR. This is an open-access article distributed under the terms of the Creative Commons Attribution License, which permits unrestricted use, distribution, and reproduction in any medium, provided the original author and source are credited.

\begin{abstract}
Background: Nurses have reported a lack of knowledge regarding substance use disorder. Previous encounters with the patient population affected by substance use disorder and a lack of knowledge can lead to a lack of perceived competency among nurses. Limited research on continuing education interventions have suggested an improvement in nurses' knowledge and sense of competency in caring for patients with substance use disorder.

Method: The purpose of this study was to evaluate a continuing education intervention on nurses' knowledge and perceived competency regarding the care of hospitalized patients with substance use disorder. A pre-test/post-test design was utilized to assess changes in knowledge and perceived competency immediately before and after the intervention for 31 hospital nurses using evidence-based tools and measures.
\end{abstract}

Results: Directly following the intervention, knowledge and perceived competency scores were significantly improved among the nurse participants, while there was no statistical significance noted between the demographic characteristics and the pre-test/post-test results.

Conclusion: The educational intervention was found to significantly improve knowledge and perceived competency scores among nurses as evidenced by the pre-test/post-test scores. Thus, nurses working with patients diagnosed with substance use disorder could benefit from continuing education on substance use disorder.

Keywords: Substance use disorder; Nursing education; Perceived competency

\section{Introduction}

Researchers have found that the competency level of nurses caring for patients with substance use disorder influence the patient's path of recovery [1]. A recent systematic review of 23 studies found that adverse attitudes of healthcare providers regarding patients with substance use disorder are common [2] and can further compound the risk of poor health outcomes in this at-risk population. Nurses who work in patient areas outside of mental health units are often under prepared to care for patients who have a secondary diagnosis of substance use disorder [2,3]. Healthcare providers may be poorly trained and ill-informed about mental illness, including substance use disorder, which may result in a lack of perceived competency [4]. A lack of knowledge and competency among nurses can be a barrier to patient's seeking treatment and long-term recovery. Substance use disorder is a growing concern as the rate of patients admitted to hospitals for issues related to substance use disorder has been increasing [5]. Although the opioid epidemic is a national problem, there is a significant prevalence among rural areas such as Appalachia [5]. Patients diagnosed with substance use disorder can be admitted to acute care units and nurses may not be prepared to effectively promote long term recovery [6]. Recent efforts have been made to address the lack of continuing education and training of nurses working with patients with substance use disorder [3].

\section{Literature Review}

Between the years from 1999 and 2016, there was a 400\% increase in death related to opioids in the United States [5]. The rate of hospital admissions and emergency room visits related to opioids increased $64 \%$ and $99 \%$ respectively [5]. In particular, rural Appalachian areas have experienced a significant increase in opioid use over the recent years [5] with higher substance use disorder rates noted in rural areas [7]. Of the rural population ages 12 years and older, $8.2 \%$ (more than 20 million people) were noted to use substances in 2013 [7]. As substance use disorder rates have increased, this disorder has become the leading cause of death in the United States [7]. Although the incidence of substance use disorder is growing, there are a limited amount of services and providers specifically designated to assist with this patient population in the rural Appalachian areas [5]. Often times, patients with substance abuse disorder face disparities including lack of insurance which causes them to utilize emergency departments for healthcare where there is more potential to be prescribed opioids than if they were being seen by a community-based primary care provider [5]. Nurses can play a vital role in assisting patients in their path to recovery by educating them about treatment and recovery resources during healthcare encounters [8].

Nurses are trained and prepared to handle medical emergencies in the hospital setting but can be ill-prepared to care for those who have substance use disorder outside of the psychiatric setting [3]. A deficiency in understanding the pathophysiology and causes of substance use disorder, including dual and secondary diagnoses, that 
are foundational in caring for patients with a history of substance use disorder is apparent [9]. The lack of nurses' knowledge and training regarding substance use can lead to a lower quality of care and safety risks for both patients and providers [9]. Furthermore, there is a lack of education regarding dual diagnoses and many nurses are unaware of how other psychiatric diagnoses can predispose a patient to substance use and how to refer patients to available resources [3].

Patients who have a history of substance use disorder may receive a lower quality of care due to nurses' lack of knowledge regarding substance use and dual diagnosis [9]. Additionally, patients who are diagnosed with substance use disorder can exhibit disruptive and aggressive behaviors while hospitalized $[6,10,11]$. These patients can be identified and perceived as unsafe by the nurses leading to altered perceptions and patient encounters which can lead to unmet health needs [12]. In addition to a lower quality of care, negative attitudes and stigma can occur due to the frustration when working with this patient population who may appear to the nurse to be drug seeking, verbally aggressive, violent or unwilling to pursue sobriety. This frustration can negatively affect the care that patients receive [13]. Furthermore, substance use disorder can predispose a patient to have violent behavior [10] which can cause nurse to fear patient encounters. A need for continuing education regarding substance use disorder and patient management has been identified [12]. Nurses in the medical-surgical setting can perceive that they lack the necessary competencies and knowledge to effectively care for the patient both physically and mentally [14].

Nursing programs lack sufficient education and training needed to prepare student nurses to care for patients with substance use disorder, contributing to the lack of knowledge among nurses [15]. Yet, following a continuing education on substance use disorder in the clinical practice setting, a survey of 489 nurses in Taiwan reported a more positive attitude. A few older studies in the United States, with smaller sample sizes of nurses, found that nurses' knowledge and confidence in caring for patients with substance use disorder were improved following an educational intervention [7,16-18]. In addition to the need for further knowledge, nurses lack confidence in their ability to de-escalate situations with substance use disorder patients and to offer treatment options which could promote quality care outcomes [6].

In a recent quasi-experimental study, a brief educational intervention to address the lack of perceived competency in caring for patients with substance use disorder was conducted for 57 nurses at a community hospital in the southeastern United States [3]. The continuing education intervention was developed and evaluated to improve knowledge and competency for nurses working with patients with substance use disorder. The evaluation consisted of a brief; fourquestion survey and 17-question knowledge quiz which assess the nurses' perceived competency and knowledge regarding working with patients with substance use disorder. There was a statistically significant improvement in each of the four survey items and improvement in the knowledge quiz scores in correlation with the educational intervention [3].

\section{Theoretical Framework}

Swanson's Middle Range Theory of Caring was utilized as the theoretical framework for this project. Swanson defined caring as nurturing another out of a feeling of obligation and accountability. Five processes were noted to influence patient care in a positive manner based on nursing interactions. These theory's concepts include: maintaining belief, knowing, being with, doing for and enabling. These concepts were utilized as the foundation for the concepts in the educational intervention to encourage the application of these concepts to patient care encounters following improved knowledge and perceived competency. The concept and attributes of caring can apply to the nurse's interaction with patients, with other nurses, and with self [18]. This theory was adapted to the context of caring for the patient with substance use disorder by promoting a positive attitude regarding the care of this at-risk patient population throughout the educational intervention [3].

\section{Methods}

\section{Sample and setting}

The study sample was comprised of nurses at a major medical center in rural Appalachia who work with patients in non-psychiatric units who have diagnoses of psychiatric disorders or substance use disorder. The hospital nurses were invited and encouraged to attend the voluntary educational course offered at various times and days to accommodate the different work schedules. Continuing education credits were approved and awarded by the Ohio Board of Nursing for participation.

\section{Design and Intervention}

A quasi-experimental design was utilized for this project with a onegroup pre-test and post-test design. The project evaluated the implementation of an educational intervention based on evidence from a previously completed educational intervention regarding nurses' knowledge and perceived competency regarding patients with dual diagnoses including substance use disorder [3]. Due to the recent publication of the study findings and the limited information on the demographic survey utilized by Russell et al. [3] there was no other hospital or study to utilize as a historical comparison group. Therefore, the one-group pre-test and post-test design was appropriate for evaluation of the educational course. The evaluation tools were administered directly before and immediately after the education course, thus it is plausible that the knowledge gained could explain the change in survey responses [19].

The materials utilized in the research study by Russell et al. [3] were shared with permission by the researchers and then reviewed by hospital management and the physician addiction specialist for approval. The educational materials and techniques included a formal instructive presentation, handouts, videos, and participant interaction. The presentation, materials, and data collection tools developed by Russell et al. [3] were adapted to incorporate the medical center's mission and vision used for this project, with permission granted by the authors [3]. Topics covered in the presentation included: psychiatric diagnoses with mention of dual diagnoses, substance use disorder, treatment options, local and community resources, recovery models, suicide risk, 12-step groups, the nurse's role in care, limit setting, de-escalation techniques and debriefing resources for nurses. The administrators and nurse leaders at the medical center also identified additional areas of need for further education for nurses working with patients who have a substance use disorder diagnosis including staff safety, limit setting and de-escalation techniques. These specific educational needs were incorporated into the instructive presentation following review and approval from administration. 
Page 3 of 5

Classes were scheduled and self-registration was completed through the hospital's online scheduling and registration system.

\section{Data collection including measures and outcomes}

A demographic survey without collection of identifying information was developed by the research team; the knowledge-based quiz and four-question perceived competency survey developed by Russell et al. [3] were used to measure outcomes. The demographic survey, knowledge-based quiz and four-question survey were administrated before the educational program to collect baseline data and the knowledge-based quiz and four-question post-test survey were administered again immediately after the educational intervention. The knowledge-based quiz consisted of 17 items based on topics covered in the educational program including substance use disorder, personality disorders, anxiety, therapeutic communication, suicide, blackout, denial as a defense mechanism, hitting rock bottom, and medications used for treatment of substance use disorder. The knowledge-based questions were in multiple choice or true/false format. The questions of the pre-test and post-test surveys were retained as published by Russell et al. [3]. The authors explained that they developed the evidence-based survey tool after discovering the lack of a relevant survey that matched their topic. The survey items were based on identified competencies to promote positive outcomes in behavioral health patients. The competences were used as guides in the development of statements which the participant could rate on a Likert scale of 1 to 5 ( 1 being strongly disagree and 5 being strongly agree) and their brief survey tool had face validity and an internal consistency reliability of 0.654 for the pre-test survey and 0.857 for the post-test survey [3].

\section{Data analysis}

Data were analyzed using SPSS ${ }^{\bullet}$ version 20 software. The primary analysis was a comparison of overall scores using descriptive statistics. The differences between the pre-test and post-test total knowledgebased quiz scores and the four survey questions were analyzed using nonparametric statistics. Wilcox on signed-rank tests were utilized by calculating the paired scores for the pre-test and post-test results and ranking the absolute differences [20].

\section{Results}

Thirty-one (31) hospital nurses completed the continuing education course and the pre-test and post-test surveys. Demographic characteristics of the participants showed that there were various types of nurses who participated ranging from licensed practical nurses (LPNs) to doctor ally prepared Registered Nurses (RNs) with the mode of registered nurses with a bachelor's degree $(38.7 \%, n=12)$ (Table 1 ). There were no statistically significant differences between the demographic factors and the responses on the knowledge quiz or survey questions.

The descriptive statistics corresponding to the knowledge quiz and survey items can be found in Table 2. Wilcox on signed-rank tests results showed significantly higher scores on the post-test scores of the knowledge-based quiz compared to the pre-test quiz $(\mathrm{Z}=-4.753$; $\mathrm{p}=0.000$ ). For the perceived competency pre-test and post-test, each of the four items were individually compared and all four survey questions were significantly higher following the intervention ( $\mathrm{Z}=-2.967$ to $-4.472 ; \mathrm{p}=0.000$ to 0.003 ). The greatest improvement was found in the participants' response to the statement regarding perceived competency in knowing the questions to ask if the nurse suspects drug or alcohol use.

\begin{tabular}{|c|c|}
\hline Variable & n (\%) \\
\hline Specialty & 31 \\
\hline Non-Clinical & $3(9.7 \%)$ \\
\hline Intermediate or Intensive Care & $3(9.7 \%)$ \\
\hline Emergency Room & $1(3.2 \%)$ \\
\hline Telemetry & $6(19.4 \%)$ \\
\hline Medical-Surgical & $6(19.4 \%)$ \\
\hline Other & $12(38.7 \%)$ \\
\hline Sex & 31 \\
\hline Male & $1(3.2 \%)$ \\
\hline Female & $30(96.8 \%)$ \\
\hline Age & 29 \\
\hline $20-30$ & $1(3 / 2 \%)$ \\
\hline $30-40$ & $5(16.1 \%)$ \\
\hline $40-50$ & $7(22.6 \%)$ \\
\hline $50-60$ & $12(38.7 \%)$ \\
\hline $60-70$ & $3(9.7 \%)$ \\
\hline Other & $1(3.2 \%)$ \\
\hline Years of Experience & 29 \\
\hline $0-5$ & $1(3.2 \%)$ \\
\hline $6-10$ & $2(6.5 \%)$ \\
\hline $11-15$ & $2(6.5 \%)$ \\
\hline $16-20$ & $9(29.0 \%)$ \\
\hline $21-25$ & $4(12.9 \%)$ \\
\hline Other & $11(35.5 \%)$ \\
\hline Education & 29 \\
\hline Licensed Practice Nurse & $2(6.5 \%)$ \\
\hline Registered Nurse with Diploma & $6(19.4 \%)$ \\
\hline Registered Nurse with Associate's Degree in Nursing & $5(16.1 \%)$ \\
\hline Registered Nurse with Bachelor's Degree in Nursing & $12(38.7 \%)$ \\
\hline Registered Nurse with Master's Degree in Nursing & $3(9.7 \%)$ \\
\hline Registered Nurse with Doctorate in Education & $1(3.2 \%)$ \\
\hline
\end{tabular}

Table 1: Demographic characteristics of hospital-based nurse participants $(n=31)$. 


\section{Discussion}

The results of this study support the findings of previous studies [3,7,16-18] and demonstrated a statistically significant improvement in hospital nurses' knowledge and perceived competency regarding caring for patients with substance use disorder following the evidence-based continuing education intervention. The current study's approach and measurement tools were based on those used by previous researchers [3], and the study findings affirm the importance of a continuing education intervention for hospital nurses regarding perceived competency in caring for this at-risk patient population. Educating nurses on substance use disorder has the potential to promote positive recovery outcomes and safety for patients and nurses [11,21].Based on the improved outcomes of the continuing education in various studies on the topic of caring for patients with substance use disorder, hospitals may consider offering such opportunities as standard nursing education requirements, especially in light of the growing rate of substance use disorder.

\begin{tabular}{|c|c|c|c|c|}
\hline Item & Pre-test average score & Post-test average score & $\mathbf{z}$ & p \\
\hline Knowledge Quiz & $78.90 \%$ & $91.70 \%$ & -4.753 & 0 \\
\hline Feel Confident to care for them and believe recovery is possible. & 3.55 & 4.23 & -3.207 & 0.001 \\
\hline Make the approach with a positive, nonjudgmental attitude. & 3.84 & 4.32 & -2.967 & 0.003 \\
\hline Know what questions to ask if I suspect drug or alcohol use. & 3 & 4.32 & -4.472 & 0 \\
\hline Refer them to resources in the community to motivate them to seek help. & 3.42 & 4.32 & -3.954 & 0 \\
\hline
\end{tabular}

Table 2: Descriptive Statistics Related to Survey Items.

Patients diagnosed with substance use disorder may have aggressive behaviors or exhibit violence $[10,11]$, possibly related to anxiety or fear that occurs during hospitalization [6]. As part of the intervention and in anticipation of the potential for aggression, de-escalation techniques for aggressive behaviors were taught in hope to deter violence before it occurs. De-escalation competencies and techniques presented during the intervention included therapeutic communication and limit setting. Nurses were also educated on how to identify signs and symptoms of agitation and aggression in patients before violence occurs. These techniques promote safety by preventing and deescalating aggression and violent behaviors in the hospital setting [6].

Patients with substance use disorder require information about post-discharge community resources to support and maintain healthy outcomes. The educational intervention provided nurses with information regarding referral to recovery resources, promoting their competence with discussing post-discharge planning with patients with a history of substance use disorder. This included resource available both inside and outside of the hospital setting. A long-term period of sobriety is the primary goal for patients with substance use disorder. Promoting sobriety requires addressing the patient's physical and psychological needs [21]. Psychological aspects of substance use disorder, such as the concept of hitting rock bottom, use of defense mechanisms, and fear of sobriety are presented and discussed in the educational intervention. Physical aspects of care include evidencebased practice pain management which, for some healthcare providers who have had negative experience in the past, may challenge nurses' sense of competency or care delivery [22]. Nurses taught how to comprehensively approach the needs of patients with substance use disorder in an evidence-based manner will be more effective at promoting recovery-focused care [21].

\section{Limitations}

Limitations included the small sample size within a single hospital, although the variety of educational levels of the nurses provided some heterogeneity in the sample. The researchers intended to conduct a delayed, second post-test survey but few nurses participated in the online post-test survey administrated one-week post-intervention, even after multiple reminders. As such, it is difficult to determine if the educational intervention had a long-term impact on nurses' knowledge and perceived competency. Future research is recommended to evaluate the long-term effect of an educational intervention using a follow-up study with incentives to retain participants.

\section{Conclusion}

Hospitalized patients with a secondary diagnosis of substance use disorder often present a challenge for nurses in non-psychiatric units. Nurses have reported a lack of knowledge and competence to care for this growing patient population in a way that promotes safe behaviors and sobriety. Nurses are in an advantageous position to promote recovery in these patients because they provide care for them on a continual basis while they are hospitalized. The results of this study indicate a statistically significant increase in knowledge and perceived competency following a continuing education intervention regarding substance use disorder among nurses working in rural Appalachia. Increasing nurses' knowledge of patients with substance use disorder through an evidence-based educational intervention, can promote safety, health, and recovery in this at-risk patient population.

\section{Acknowledgement}

My sincere appreciate to Dr. Marjorie Vogt and Dr. Kimberlee Miller-Wenning for their feedback. Thank you to Dr. Jo Andrea Watson and Dr. Joey Traderand the medical center administrators and nurse leaders for supporting this project.

\section{References}

1. Zolnierek CD (2009) Non-psychiatric hospitalization of people with mental illness: systematic review. Journal of Advanced Nursing 65: 1570-1583.

2. Van Boekel L, Brouwers E, Van Weeghel J, Garretsen H (2013) Stigma among health professionals towards patients with substance use disorders 
Citation: Perry NR, Chertok IRA (2018) Continuing Education Model for Nurses Regarding Substance Use Disorder. J Health Educ Res Dev 6: 272. doi:10.4172/2380-5439.1000272

Page 5 of 5

and its consequences for healthcare delivery: Systematic review. Drug and Alcohol Dependence 131: 23-35.

3. Russell R, Ojeda MM, Ames B (2017) Increasing RN perceived competency with substance use disorder patients. The Journal of Continuing Education in Nursing 48: 175-183.

4. Kassam A, Papish A, Modgill G, Patten S (2012) The development and psychometric properties of a new scale to measure mental illness related stigma by health care providers: The Opening Minds Scale for Health Care Providers (OMS-HC). BMC Psychiatry 12: 62.

5. Rigg KK, Monnat SM, Chavez MN (2018) Opioid-related mortality in rural America: Geographic heterogeneity and intervention strategies. International Journal of Drug Policy 57: 119-129.

6. Rutledge D, Wickman M, Cacciata M, Winokur E, Loucks J, et al. (2013) Hospital staff nurse perceptions of competency to care for patients with psychiatric or behavioral health concerns. Journal for Nurses in Professional Development 29: 255-262.

7. Puskar KR, Heeyoung L, Mitchell AM, Kane I, Albrecht SA, et al. (2016) Inter professional collaborative education for substance use screening: Rural areas and challenges. Online Journal of Rural Nursing \& Health Care 16: 76-96.

8. Leahy LG (2017) The opioid epidemic: What does it mean for nurses? Journal of Psychosocial Nursing and Mental Health Services 55: 18-23.

9. Kelleher S, Cotter P (2009) A descriptive study on emergency department doctors' and nurses' knowledge and attitudes concerning substance use and substance users. International Emergency Nursing 17: 3-14.

10. Lim JY, Lui CK (2016) Longitudinal associations between substance use and violence in adolescence through adulthood. Journal of Social Work Practice in the Addictions 16: 72-92.

11. Sinani L, Kola V, Elezi F, Cenko F (2016) The effect of alcohol and substance on human aggression. International Journal of Ecosystems \& Ecology Sciences 6: 203-206.
12. Peckover S, Chidlaw RG (2007) Too frightened to care? Accounts by district nurses working with clients who misuse substances. Health \& Social Care in the Community 15: 238-245.

13. Ford R (2011) Interpersonal challenges as a constraint on care: The experience of nurses' care of patients who use illicit drugs. Contemporary Nurse: A Journal for the Australian Nursing Profession 37: 241-252.

14. Neville K, Roan N (2014) Challenges in nursing practice: Nurses perceptions in caring for hospitalized medical-surgical patients with substance abuse/dependence. The Journal of Nursing Administration 44: 339-346.

15. Chang Y, Yang M (2013) Nurses' attitudes toward clients with substance use problems. Perspectives in Psychiatric Care 49: 94-102.

16. Graham AV, Christy K, Emmitt-Myers S (1997) Substance abuse education for clinical nurses: a controlled study. Journal of Continuing Education in Nursing 28: 217-222.

17. Gerace LM, Hughes TL, Spunt J (1995) Improving nurses' responses toward substance-misusing patients: A clinical evaluation project. Archives of Psychiatric Nursing 9: 286-294.

18. Marcus MT, Rickman KA, Sobhan T (1999) Substance abuse education liaisons: A collaborative continuing education Program for nurses in acute care settings. Journal of Continuing Education in Nursing, p: 229.

19. Polit DF, Beck CT (2012) Nursing research: Generating and assessing evidence for nursing practice. 9th edn, pp: 197-203.

20. Amendolair D (2012) Caring model: Putting research into practice. International Journal for Human Caring 16: 14-21.

21. Lim E, Wynaden D, Heslop K (2018) Changing practice using recoveryfocused care in acute mental health settings to reduce aggression: A qualitative study. International Journal of Mental Health Nursing.

22. Krokmyrdal KA, Andenaes R (2015) Nurses' competence in pain management in patients with opioid addiction: A cross-sectional survey study. Nurse Education Today 35: 789-794. 